\title{
Zea mays L. Pollen: An Approach to Its Quality Control
}

Maria Graça Campos ${ }^{1}$, Norma Almaraz-Abarca ${ }^{2}$, Miguel Pires Matos ${ }^{1}$, Nelson Mortágua Gomes ${ }^{1}$, Vanilda Aparecida Arruda ${ }^{3}$, Ortrud Monika Barth ${ }^{4}$, Alex Silva Freitas ${ }^{4}$, Débora Cristina Amâncio ${ }^{1}$ and Lígia Bicudo Almeida-Muradian ${ }^{3}$

1. Coimbra Chemistry Center, Faculty of Sciences and Technology, and Faculty of Pharmacy, University of Coimbra, Polo III, Azinhaga de Santa Comba, Coimbra 3000-548, Portugal

2. Interdisciplinary Research Centre for Integral Regional Development-Durango, National Polytechnic Institute, Sigma 119, 20 de Noviembre II, Durango 34220, DGO, México

3. Department of Food and Experimental Nutrition, Pharmaceutical Science School, University of São Paulo, Av. Prof. Lineu Prestes 580, Bloco 14, CEP055800-000, São Paulo, Brazil

4. Instituto Oswaldo Cruz, Fundação Oswaldo Cruz, Avenida Brasil 4365, Rio de Janeiro 21040-900, Brazil

\begin{abstract}
Zea mays L. is one of the biggest cropping systems among the sustainable development agronomy. Pollen from this crop source is unexplored and apiculture can be a good partner adding value to the product and creating new jobs helping to solve some social issues as unemployment. However, food safety is crucial, thus the aim of this study was to explore the flavonoid/phenolic profiles from Z. mays L. pollen as a fingerprint for this plant identification and also to demonstrate how the method of bee pollen samples (honeybee collected pollen) is applied. For this purpose, several sources of Z. mays L. pollen were analyzed, including corn hybrids and genetic modified samples collected at the breeding fields. For this work, samples were taken at several years from 2000 to 2012 and collected from different countries and locations, such as Portugal, Mexico and Brazil. Results showed, for the first time, that the fingerprint (flavonoid/phenolic profile) for Z. mays L. pollen does not change over the time of sampling neither with the region of harvesting. The high performance liquid chromatography-diode array detector (HPLC/DAD) fingerprints of phenolic/flavonoid extract from Z. mays remain unchanged for all samples analyzed from different countries, hybrids and/or genetic modified plants. This is also the first study reporting these phenolic compounds not only in pollen collected directly from hybrid plants, but also in $Z$. mays bee pollen. The described fingerprinting method is easy, fast and accurate for the characterization of $Z$. mays L. pollen samples and complete microscopic analysis because it is species-specific.
\end{abstract}

Key words: Flavonoids, phenols, Zea mays, pollen, bee pollen, food safety, phenolic fingerprinting, palynological analysis, oxygen radical absorbance capacity (ORAC).

\section{Introduction}

Zea mays L. is one of the world's most important crops. It is an annual grass from the Poaceae family that grows rapidly in fertile lands [1]. The huge amount of pollen could be used adding value to this product.

An easy way to get pollen from flowers in high level production is the use of honeybees to make the collection. These mixtures of pollen vary widely in composition, but when the bees harvest the pollen in

Corresponding author: Maria Graça Campos, professor, research fields: pharmacognosy and drug discovery. breeding crops, the product could be monofloral [2] as the example of Zea mays bee pollen. The crude material is composed by pollen grains collected by honey bees in different plant species, depending on the local flora availability, and it is estimated that in certain regions, the monofloral collection can achieve various tones of this crude material, as exemplified by Helianthus annus L. (France), Cistus ladaniferus L. (Spain) and Cocos nucifera L. (Brazil) pollen [3-5].

Beekeepers collect this crude material to be sold as food (fresh or dried) [2]. Despite the main interest in the quality control of this product, proper identification prior taxonomic studies are also relevant. 
The recent development of corn varieties not only for industrial purposes, but also for pharmaceutical applications, turns corn and its pollen as attractive targets for research [6]. Consequently, the bee pollen from Z. mays L. is a potential source for the development of pharmaceuticals and nutraceuticals.

Several studies have been reported on the chemical composition of corn pollen. For instance, regarding to sugars, sucrose comprises $98 \%$ of the soluble carbohydrates and $5 \%$ of pollen fresh weight. For the remaining sugar portion, the amount of fructose and glucose is inversely proportional to sucrose depending on the storage conditions. Proline is the most abundant free amino acid, but also serine and alanine exist in appreciable quantities. Pollen is also the corn tissue with the greatest concentration of sterols, with a chemical profile mainly represented by 24(28)-methylene-sterols and small amounts of castasterone and other brassinosteroids [7].

In addition to lipids and proteins, small aromatic molecules, such as carotenoids, jasmonates (lipid-derived compounds), flavonoids, phenolic acids and most of the classic phytohormones, have been detected in pollen. From all those compounds, only flavonols, a specific class of flavonoids, have a demonstrated a role in pollen germination [7]. Virtually, all pollens accumulate flavonoids, often to very high levels. Although flavones are absent from corn pollen, particular forms of flavonol glycosides are stored and seem to be exclusive from this reproductive structures [8]. This glycosides are mainly derived from kaempferol, quercetin and isorhamnetin [9], and the quercetin-3,3'-O-diglucoside is identified as the most abundant in Z. mays L. pollen [7].

Despite some previous reports on the flavonoid/phenolic content of corn pollen $[8,10]$, to the best of our knowledge, there is no research on $Z$. mays L. pollen flavonoids profile in hybrid or genetic modified cultivars as well as in corn bee collected pollen. Thus, the aim of this study was to characterize flavonoid/phenolic profiles from $Z$. mays L. pollen, from hybrids and genetic modified plants, as a fingerprinting reference for both plant and bee pollen.

\section{Materials and Methods}

\subsection{Materials}

Portuguese hybrid samples were provided by Silas Pêgo from the Agronomic Station of Braga Germoplasm Centre (Centro de Gestão de Vale do Sousa) and belonged to an extend project including free pollination- "population improvement" and "recurrent selection", for national add value genetic resources; other Portuguese corn pollen samples were collected in Coimbra from corn cultivar fields with Anjou hybrids No. 246 (2000-2002).

The genetically modified corn pollen samples used in this study were collected in breeding fields from Vale do Mondego, Coimbra, in 2009. Mexican corn pollen and bee pollen samples were gathered in Durango in 2000. Brazilian bee pollen samples were collected in São Paulo and Mato Grosso state, in 2011.

Samples of Z. mays L. pollen loads from bee pollen mixtures, were firstly separated by color $[11,12]$ and selected for microscopic and high performance liquid chromatography-diode array detector (HPLC/DAD) analysis in order to obtain its monofloral origin.

Z. mays L. pollen, purchased from Sigma, was analyzed as a standard reference.

\subsection{Methods}

\subsubsection{Preparation of Pollen for Microscopy}

Bee pollen was processed according to the methods described in Ref. [13]. Dry pollen loads of $2 \mathrm{~g}$ of a sample (this is a pool of circa 300 pollen loads) were mixed with $70 \%$ ethanol to complete $13 \mathrm{~mL}$, and sonicated for $5 \mathrm{~min}$. The sediment obtained after centrifugation $(1,500 \mathrm{rpm} / 15 \mathrm{~min})$ was extracted with ethanol, and sonicated again. A solution of distilled water/glycerin 1:1 was added to the sediment to complete $13 \mathrm{~mL}$, and left for about $30 \mathrm{~min}$. 


\subsubsection{Preparation of Microscope Slides}

One drop of the well-mixed pollen grain suspension was applied on a microscope slide, covered with a cover slide and sealed with enamel. The slides were maintained in a horizontal position for $10 \mathrm{~d}$ or more before start to dryness.

\subsubsection{General Experimental Procedures}

\subsubsection{Sample Preparation}

$10 \mathrm{mg}$ of dried pollen of each sample were sonicated in an ethanol-water solution $(1 \mathrm{~mL}, 50 \% \mathrm{v} / \mathrm{v})$ for 60 $\min$. The resultant mixtures were centrifuged at 5,000 rpm for $10 \mathrm{~min}$, and the supernatants were used for HPLC/DAD analysis as previously described in Refs. $[11,13]$ and for oxygen radical absorbance capacity (ORAC) assays as described in Ref. [14].

\subsubsection{HPLC/DAD Analysis}

Extracts $(20-50 \mu \mathrm{L})$ were analyzed on a Gilson 170 and Waters Spherisorb ODS2 $(5 \mu \mathrm{m})$ column $(4.6 \mu \mathrm{m}$ $\times 250 \mathrm{~mm}$ ) by an acidified acetonitrile-water gradient at $0.8 \mathrm{~mL} / \mathrm{min}$ flow rate, and the column temperature stated at $24{ }^{\circ} \mathrm{C}$. Standard chromatograms were plotted at $\lambda_{\max }=260-340 \mathrm{~nm}$. These wavelengths were chosen, because flavonols and flavones, the main compounds in pollens, have two characteristic bands of ultraviolet absorption close to these values. Spectral data for all peaks were accumulated in the range of $\lambda_{\max }=220-400$ $\mathrm{nm}$ (the phenolic/flavonoid spectra are all in this display) using DAD (Gilson 170). The apparatus are HPLC system (Gilson) and DAD with Unipoint software, and Jasco V-530 UV/VIS spectrophotometer with "Spectra Manager" software.

The phenolic profile of each sample was made up of all compounds resolved in its respective chromatogram. The structural information of each compound was made by direct comparison of retention time and ultraviolet absorption spectra according to the information compiled by Campos and Markham [15].

\subsubsection{Isolation of Z. mays L. Pollen Compounds}

The isolation of flavonoids was carried out with bee pollen samples and used as markers for the other source of pollen used in the assays. Pellets of species-specific pollen were fragmented in EtOH: $\mathrm{H}_{2} \mathrm{O}$ (1:1) with ultrasound, and the dispersed pollen grains left to extract overnight. The extracts were separated by thin layer chromatography (TLC) using Whatman chromatography paper 3MM. The extracts were submitted to centrifugation before application on sheets of Whatman 3MM chromatography paper for one dimension separation. As development solvent, $15 \%$ of aqueous acetic acid was used. Each compound was prepared for further study by chromatography on a $\mathrm{C}-18$ reversed phase column using water-acetonitrile gradient and checked for purity by HPLC/DAD according to Markham and Campos [16].

2.2.3.4 Nuclear Magnetic Resonance (NMR) Analysis

For the full structure elucidation, NMR spectra were carried out in Bruker AC apparatus at $300 \mathrm{MHz}$ with isolated compounds solved in $\mathrm{DMSO}^{-\mathrm{d}_{6}}$ and with tetramethylsilane (TMS) as internal standard at $30{ }^{\circ} \mathrm{C}$ [17].

\subsubsection{ORAC Assay}

The ORAC procedure used an automated plate reader (Synergi HT, Bio TeK, USA) with 96-well plates $[14,18]$. Analyses were conducted in a $\mathrm{pH} 7.4$ $(75 \mathrm{mM})$ phosphate buffer at $37{ }^{\circ} \mathrm{C}$ under condition with a blank sample in parallel. The extracts were diluted with sodium phosphate buffer $(75 \mathrm{mM}, \mathrm{pH}$ 7.4). Peroxyl radical was generated using 2, 2'-azobis (2-amino-propane) dihydrochoride (AAPH) aqueous solution, which was prepared for each run $(153 \mathrm{mM})$. Fluorescein was used as substrate $(40 \mathrm{nM})$. The ORAC analyzer was programmed to record the fluorescence of fluorescein every minute after addition of AAPH. All fluorescent measurements were expressed relatively to the initial reading. Fluorescence analysis conditions were performed as follows: excitation at $493 \mathrm{~nm}$ (filter 485/20) and 515 $\mathrm{nm}$ (filter 528/20). The standard curve was linear between $6.25 \mu \mathrm{M}$ and $100 \mu \mathrm{M}$ Trolox ${ }^{\circledR}$. The final results were calculated using the differences of areas under the fluorescence decay curves between the 
blank and a sample, and were expressed as $\mu \mathrm{M} \mathrm{TE} / \mathrm{g}$ bee pollen.

\section{Results and Discussion}

To get the maximum commercial value and quality of bee pollen to be exploited as a nutraceutical or as a vegetal matrix with therapeutic value, it is important to define characteristics that can be used as a passport, which will define their identity (ID) in various countries.

To contribute to this ID of Z. mays L. bee pollen, in this work, it was carried out with microscopic analysis and HPLC/DAD fingerprints supplemented with ORAC analysis. Still missing macro- (proteins, carbohydrates and lipids) and micro-nutrients (as vitamins, minerals, etc.), they will be included in the passport to complete the full data.

\subsection{Microscopic Analysis}

As a start point, the microscopic analysis provides the first approach to the identification of the pollen floral origin. The main characteristics, as size, shape and ornamentation compared with the available data published and the pollen hand collected from the plant, will provide enough information to complete this step.

As an example, images of fresh Z. mays L. pollen grains are presented in Fig. 1. They are spheroidal with a smooth surface (exine) and present one porus

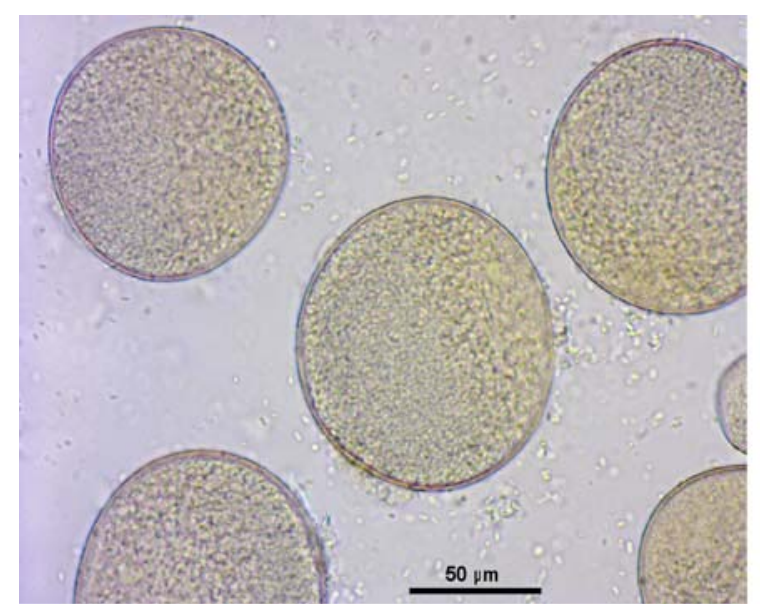

provided with an annulus. Its content was mainly composed of amyloplasts.

\subsection{HPLC/DAD Analysis}

General HPLC/DAD profile (fingerprint of the phenolic and polyphenolic compounds, including flavonoids) obtained with the samples is presented in Fig. 2. Samples, as Anjou hybrid No. 246 from Portugal and Mexico, bee pollen from Durango (Mexico) and from the states of São Paulo and Mato Grosso (Brazil), show the same fingerprint of the flavonoid profile by HPLC/DAD. Other samples displaying the same flavonoid profile are the entire 1st group-Pigarro assay (smooth white grain)/Portugal, the 2nd group - three simple hybrids (HS's)/Portugal, the 3rd group-Azores regional corn/Portugal and the 5th group- “Amiúdo" corn (smooth yellow grain) from Portugal obtained by the project "Population Improvement" and "Recurrent Selection" for national add value genetic resources.

The seven flavonoids shown in Fig. 2 are used as chemical markers for this $Z$. mays L. pollen as reported in a previous paper [8], where they study the phenolic composition of 32 populations of $Z$. mays $L$. subsp. mexicana, Z. mays L. subsp. parviglumis, $Z$. mays L. subsp. mays, Z. diploperennis, Z. perennis and $Z$. luxurians from Mexico and Guatemala.

The ultraviolet theory developed by Campos and

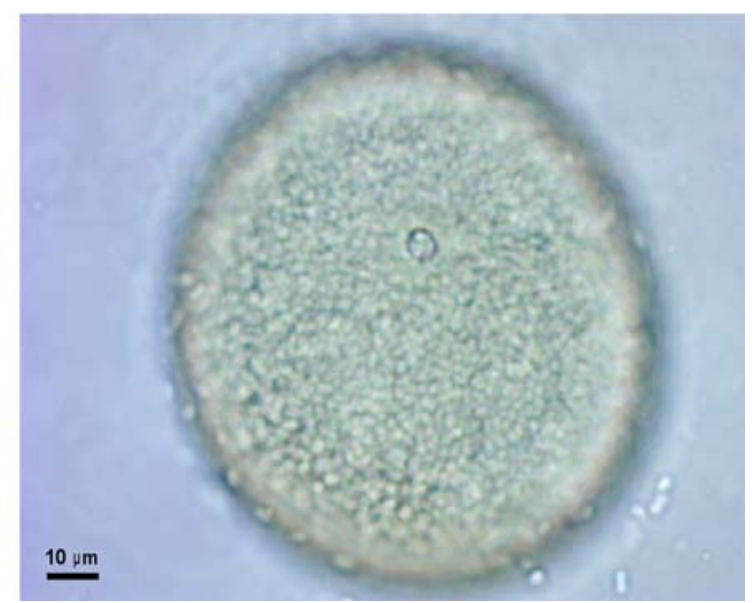

Fig. 1 Z. mays L. pollen grains.

The right side pollen grain at a higher magnification, presents its unique porus with a smooth annulus. Amiloplasts are visible inside and dispersed between the pollen grains ((left side of the figure). 


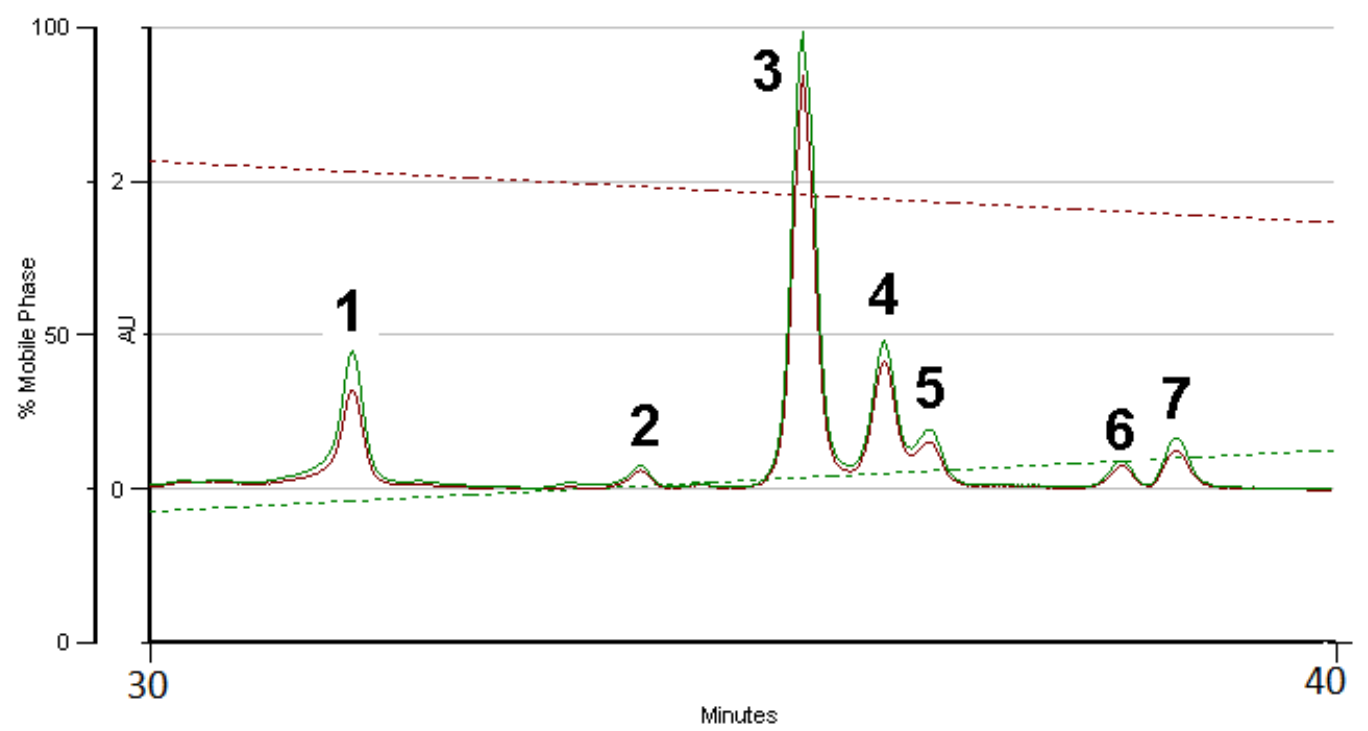

Fig. 2 HPLC/DAD $(\lambda=260-340 \mathrm{~nm})$ profile of flavonoid extract from $10 \mathrm{mg} \mathrm{Z}$. mays $\mathrm{L}$. pollen/1 $\mathrm{mL}$ ethanol of $50 \%$ (injection $50 \mu \mathrm{L}$ ).

Solvents: water $\mathrm{pH} \sim 2.2$ with $o$-phosphoric acid (solvent $\mathrm{A}$ ) and acetonitrile (solvent $\mathrm{B}$ ).

Solvent gradient profile: time $(\mathrm{T})=0$, acetonitrile $(\mathrm{B})=0 \% ; \mathrm{T}=12, \mathrm{~B}=9 \% ; \mathrm{T}=20, \mathrm{~B}=13 \% ; \mathrm{T}=40, \mathrm{~B}=33 \% ; \mathrm{T}=45, \mathrm{~B}=33 \%$.

Flow rate: $0.8 \mathrm{~mL} / \mathrm{min}$.

Markham [15] was a precious help to draw the first approach to the flavonoid structures of those compounds. They were then isolated by paper chromatography, and the NMR analysis finalized the information on the structures, confirming the previous hypothesis proposed by the Campos and Markham [15] and the data published in the literature.

Those rules applied to these compounds involve predetermined evidence as explained in the following. The ultraviolet spectra presented two clear bands of absorption, near $\lambda=250 \mathrm{~nm}$ (band II) and $\lambda=350 \mathrm{~nm}$ (band I), showing that the main structure was a flavone and/or flavonol-3-derivative. To distinguish them, the intensity on the absorption was used. If band I is more intense than band II in general, it is a flavone (C3 with hydrogen); and if it is the inverse, band I less intense than band II, it is a 3-substitued flavonol but different from a free $\mathrm{OH}$. The substituents at $\mathrm{C} 3$ in these cases are mainly glycosides, but phenolic acids can also be the substituents. In this last situation, band I will be higher than band II and the shape will be similar to the phenolic acid itself.

Other important rule comprises the shape of band II
( $\lambda$ between $250 \mathrm{~nm}$ and $285 \mathrm{~nm}$ ). When flavones or flavonols lack B-ring substitution, or possess a 4'-oxygenated B-ring, band II of the spectrum appears as only one peak (for example, apigenin have a maximum absorption at $\lambda=267 \mathrm{~nm}$ and kaempferol-3-O-derivatives with $\lambda=265 \mathrm{~nm}$ ). The introduction of additional B-ring oxygenation commonly produces a double band II (bands IIa and IIb). Band IIa may appear simply as a shoulder on the long wavelength side of band IIb, for example, luteolin and quercetin-3-O-derivatives with the present maximum of absorption at $\lambda=254-255 \mathrm{~nm}$ and a shoulder at $266-267 \mathrm{~nm}$. If it is the inverse situation, Band IIa appears more intense and the shoulder on wavelength side of band IIb, a possible substitution at C6 or C8 (A-ring) or at C3' (B-ring) are the more probably possibilities [15].

If an exact match for the spectrum can be found, but the retention time (RT; min) differs, then it is likely that the unknown diverges from the reference only in the number or nature of sugars attached to the glycosylated site, or in the nature of a substituent, e.g., $O$-methyl compared with $O$-ethyl, or presence/absence 
of an aliphatic acyl group on a sugar. If the unknown is eluted faster than the reference (i.e., has a lower $\mathrm{RT}$ ), then because the chromatographic solid phase is non-polar relative to the polar solvent, this indicates that the unknown is more polar (e.g., contains more sugars or a sugar of higher polarity). Conversely, if the unknown is eluting slower than the reference (i.e., higher RT), then the unknown is less polar (e.g., contains fewer sugars or a sugar of lower polarity or contains an aliphatic acyl group). In the event that no exact match for the absorption spectrum can be found amongst the reference spectra, a close match may indicate that the unknown is related to the reference, perhaps with the same oxygenation pattern but with a different substitution pattern. If the spectra present a shoulder between $280-310 \mathrm{~nm}$, a free $7-\mathrm{OH}$ is possible, and by the contrary if this does not exist, it could be caused by a 7-O-glycosilation [15].

With regard to compound identification, a reliable proof of identity of an unknown with a reference compound requires comparison via a minimum of three characters. RT and an absorption spectrum comprise only two, and ideally any identification should include one other technique as well, for example, TLC, or a mass spectrum or a NMR spectrum as was carried out in this work.

Following these rules, the ultraviolet spectra of the compounds provided by the HPLC/DAD obtained with the hidroalcoholic extract of Z. mays L. pollen gave several quercetin derivatives. The main, were flavonols identified as quercetin-3,7-O-glucoside (compound 1), quercetin-3,7,3'-O-diglucoside (compound 2) and quercetin-3,3'-O-diglycoside (compound 3). These data are consistent with the previous reports by other authors [9].

From the ultraviolet (UV) spectral information, compound $1(\mathrm{RT}=31.68 \mathrm{~min})$ with $\lambda_{\max }=254,265 \mathrm{sh}$ and $350 \mathrm{~nm}$ (where $254 \mathrm{~nm}$ and $350 \mathrm{~nm}$ is the maximum of absorption for band I and band II, respectively, and $265 \mathrm{sh}$ is a shoulder in band II that correspond to band IIa), compound $5(\mathrm{RT}=36.69 \mathrm{~min})$ and compound 7 (RT $=38.69 \mathrm{~min}$ ) are quercetin-3-O-glycosides. The absorption at $\lambda=254$ $\mathrm{nm}$ and a shoulder at $\lambda=265 \mathrm{~nm}$, is a typical oxygenation pattern in B-ring at 3', 4', and band I with a $\lambda=350 \mathrm{~nm}$ and lower than band II corresponds to 3-O-derivatives. However, compound 1 differs from the other two in the absence of shoulder absorption $\lambda$ between $280 \mathrm{~nm}$ and $310 \mathrm{~nm}$, meaning that $\mathrm{C} 7$ is substituted, corresponding in this case to quercetin-3,7-O-glucoside. Comparing the retention times and the NMR data, compounds 6 and 7 are quercetin-3-O-sophoroside and quercetin-3-O-glucoside, respectively.

Compound $2(\mathrm{RT}=34.06)$ and $3(\mathrm{RT}=35.4)$, are also quercetin-3-O-glucosides, but with other substitutions in addition to the compounds mentioned above. They keep the main absorption points at $\lambda=$ 254, 265 and $350 \mathrm{~nm}$, but now with an inversion at the intensity in band IIb and IIa, $\lambda_{\max }=254 \mathrm{sh}, 265$ and $350 \mathrm{~nm}$ (where the maximum of UV absorption in the spectrum are the point $265 \mathrm{~nm}$ and $350 \mathrm{~nm}$, corresponding to band IIa and band I, the shoulder is located at $254 \mathrm{~nm}$ correspond to band IIb), seen in spectra 2, 3 and 4 of Fig. 3. This provides the information that a substitution at $\mathrm{C} 6, \mathrm{C} 8$ or $\mathrm{C} 3$ ' can occur, being the main difference that implies the decrease polarity (increasing the retention time) in the sugar residue. From all the analysis carried out, this compound was identified as quercetin-3-O-glucoside-3'-O-diglucoside.

Compound $3(\mathrm{RT}=35.4 \mathrm{~min})$ with an ultraviolet spectra with $\lambda=254 \mathrm{sh}, 265$ and $350 \mathrm{~nm}$, was detected as the main flavonoid in all extracts. From the reading of these values, it was clear that the decrease of UV absorption of band I related to the intensity of band II indicates a possible substitution in $\mathrm{C} 3$ at $\lambda_{\max }=350$ $\mathrm{nm}$, as already referred for the previous spectra. The appearance of two points of absorption in band II (IIb $=254 \mathrm{sh}$, IIa $=265 \mathrm{~nm}$ ) is indicative that in the B-ring exist a double substitution usually in 3' and 4'. From the chemical shift made with the isolated compound 
and boric acid (for the $\mathrm{C} 7$ hydroxide) and sodium methoxyl (for C4' hydroxide), the shift occurred in both $\mathrm{A}$ and $\mathrm{B}$ ring. The proposed structure, quercitin-3-O-glucose-3'-glucoside, is line with other authors cited above in the text.

Compound $4\left(\mathrm{RT}=36.19 ; \lambda_{\max }=252.6,265.5\right.$ and $343.8 \mathrm{~nm})$, and compound $6\left(\mathrm{RT}=38.18 ; \lambda_{\max }=254\right.$, $265 \mathrm{sh}$ and $353 \mathrm{~nm}$ ), are isorhamnetin-3-O-glycosides. Compound 4 present both $\lambda_{\max }=252.6 \mathrm{~nm}$ and 265.5 $\mathrm{nm}$ with the same intensity of absorption, however between them an intense valley is observed and this is generally indicative of a methoxy-substitution. The increased intensity of band IIa to the same value of band IIb, is induced by the glycosidic substitution at 4'. In this case, the compound was isorhamnetin-3-O-glucoside-4'-O-diglycoside.

In this case, comparing values present in this paper with the authors' previous published data, the compound could be identified as isorhamnetin-3-O-diglycoside, and suggested neohesperidoside as the dyglycoside. In Ref. [4], the authors presented this isolated compound from maize pollen, as phagostimulant in Z. mays pollen.

Compound $5(\mathrm{RT}=36.69)$ and compound $7(\mathrm{RT}=$ 38.69) are quercetin-3-O-dyglycoside and quercetin-3-O-glucoside, respectively, absolutely in line with Ref. [9] with the ultraviolet spectra, presenting the same shape and corroborating the same oxygenated pattern and substitution at $\mathrm{C} 3$ [15].

Compound 6 is an easy ultraviolet spectra, similar to many others described in the literature for isorhamnetin-3-O-glycosides [15] and after acidic hydrolysis, the aglycone reveals to be free isorhamnetin.

The spectra and structure of the compounds separated by HPLC/DAD are presented at Fig. 3 and corroborate the data explained above, based on this UV and NMR results in comparison with literature on known corn pollen and other floral flavonol glycosides.

As discussed above, this crude material can be explored with various purposes. One of them can be related to therapeutic applications. Kim and Mullin [7] cited a patent made with pollen flavonoids as active components for a plurality of functions for promoting health. The invention includes an extract of Typhae pollen prepared by a plurality of extraction processes, which will be applied to create a medicine [19].

The complete analysis of all the cited Z. mays L. pollen sources (different hybrids, transgenic and non-trangenic samples, plants from various countries breeding at different years of harvesting) confirms the previous published data that the low level of variability of the pollen phenol profiles throughout the genus Zea supports the proposal that maize and some teosintes are conspecific groups, and also indicates that the pollen phenol composition is highly conserved in the different taxa of Zea [8]. This information allows the use of the profile shown in Fig. 2 for the passport/ID (identification) of $Z$. mays L. bee pollen for commercial purposes as suggested at the beginning of this paper.

\subsection{ORAC Analysis}

The ORAC was only carried out with fresh samples (two Brazilian samples) and can be used as preliminary information for further studies involving the full quality control of the product. Free scavenging bioactivity was already carried out with DPPH given an $\mathrm{EC}_{50}=7.42 \mu \mathrm{g} / \mathrm{mL}$ by Chantarudee et al. [20] that is near the authors' previous results, $\mathrm{EC}_{50}=10.3$ $\mu \mathrm{g} / \mathrm{mL}$ in a mixture with a high prevalence of $Z$. mays L. [10].

The results of antioxidant activity by ORAC method were between $142.66 \pm 8.02 \mathrm{TE} / \mathrm{g}$ and 232.40 $\pm 6.02 \mathrm{mM} \mathrm{TE} / \mathrm{g}$ of bee pollen. Arruda et al. [14] determined the antioxidant activity of bee pollen from Cocos nucifera sp. and found values between 132.98 $\mathrm{mM} \mathrm{TE} / \mathrm{g}$ and $575.85 \mathrm{mM} \mathrm{TE} / \mathrm{g}$ of dehydrated bee pollen. Another reference was the folder from Brunswick Laboratory (October 16, 2002), citing results identical to those determined in pollen (not specified if it was dehydrated). The laboratory compared the data obtained for the antioxidant activity 

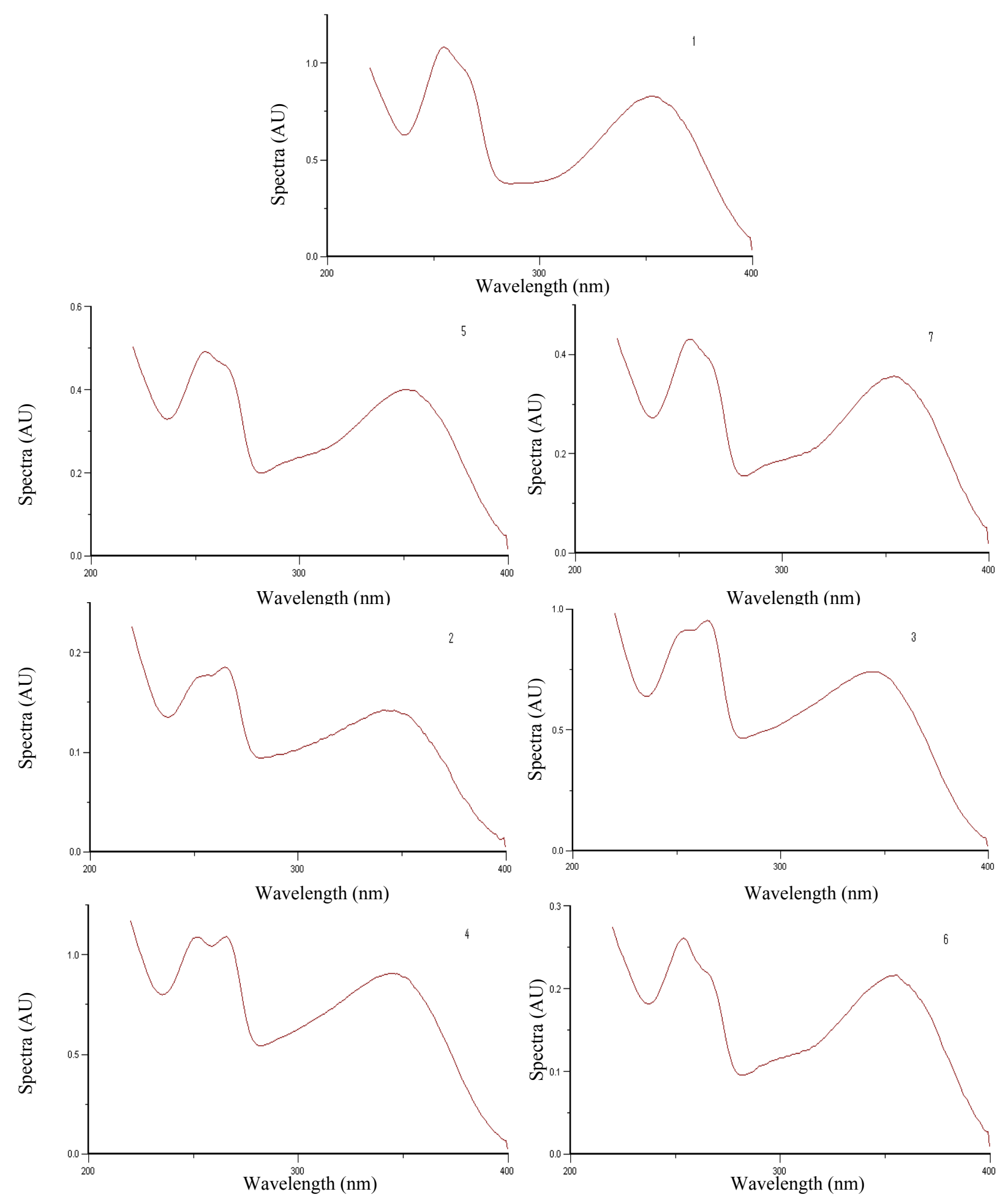

Fig. 3 Ultraviolet spectra of the of flavonoid extract of Z. mays L. pollen obtained on-line in the HPLC/DAD method [12, 15].

Compounds 1, 5 and 7 present a typical quercetin-3-O-substitution and the absence of the shoulder between 280-310 nm is indicative of the 7-substitution; compounds 2 and 3 are the quercetin-3, 3'-derivatives, presenting the same UV-spectra but with different retention time; compound 4 is a unique isorhamnetin-3-O-glucoside-4'-O-diglycoside structure, with the same intensity of absorption for both band IIa and IIb; compound 6 is a typical isorhamnetin-3-O-glycoside where the only difference from quercetin-3-O-glycoside is in the shape of band IIa and $\mathrm{Ib}$, that in this case is more accentuated. 
of the High Desert ${ }^{\circledR}$ bee pollen from CC Pollen Co. with the values of other foods. It is for the High Desert ${ }^{\circledR}$ bee pollen a value of $247 \mathrm{mM}$ TE/g bee pollen (High Desert Bee). Among the other foods, the highest value was obtained for black raspberry $(164 \mathrm{mM} \mathrm{TE} / \mathrm{g}$ sample), followed by pomegranate (105 mM TE/g sample). The results for the bee pollen samples from $Z$. mays sp. were higher than the majority of foods referenced in USDA first database of antioxidant activity for 277 selected foods using ORAC methodology, and comparable to chocolate products derived from the same table.

The bioactivity shown in the ORAC is important to reveal the freshness of the compounds for further studies as start point to evaluate the state of conservation of the matrix. Nevertheless, they could also give important information for the development of research correlated to diseases that are caused by free radicals. It is known that the antioxidant activity itself is not enough to cure those situations, but could be good startup information about the potential of some of these molecules. Until there, consumers eat the dry product and the freshness during the transport could be done using this method or even DPPH [21, 22].

\section{Conclusions}

The results showed that it was obtained the same profile for all the samples, indicating that the fingerprint (flavonoid/phenolic profile) for Z. mays L. pollen is independent from the collection sites and species (hybrids and transgenic). It is the first time that these data are published and this is also the first study reporting these phenolic compounds not only in pollen collected directly from various hybrid plants but also in Z. mays bee pollen.

The described fingerprinting method based on the flavonoid/phenolic profile is an extremely useful tool for the characterization of corn pollen, and proved to be a unique and potent methodology that could support and substitute the microscopic analysis.
Further studies will be developed with the main metabolic groups as proteins and aminoacids, glucides, lipids, vitamins and minerals to complete the full passport of the characteristics of Z. mays L. bee pollen.

At this stage of the studies, it is possible to conclude that pollen phenolic profile of maize is a highly conserved chemical marker in the different races and varieties from different countries, since they have essentially the same profile, in spite of the intensive genetic manipulation that men have carried out on the species for thousands of years, and also in spite of the recent but impressive transgenic manipulation, which has not impact on this chemical markers. That conservation can be observed not only in maize (Z. mays subsp. mays) but in all other taxa forming the genus Zea (teosintes), as it has been corroborated in the discussion above.

\section{Acknowledgments}

The authors are grateful to "Strategic Project RG-Centre-177-3717 from Science and Technology Fundation”, UID/QUI/00313/2013 and POCI-01-0145-FEDER-007630 (Portugal); to Engineer Silas Pêgo for providing the samples, from its project at the Agronomic Station of Braga Germoplasm Centre (Centro de Gestão de Vale do Sousa); to FAPESP and CNPq for financial support (Brazil); to Comisión de Operación y Fomento a las Actividades Académicas, Instituto Politécnico Nacional (Mexico) for the stimuli for research. Also they appreciate Fundação para a Ciência e Tecnologia (FCT) for the scholarship (SFRH/BD/65671/2009) supported by the European Social Fund (ESF).

\section{References}

[1] Salazar-Sosa, E., Trejo-Escareño, H. I., Vázquez-Vázquez, C., and López-Martínez, J. D. 2007. "Corn Production under Subsurface Drip Irrigation and Application of Cow Manure." International Journal of Experimental Botany 76: 169-85.

[2] Campos, M. G., Bogdanov, S., Almeida-Muradian, L. B., 
Szczesna, T., Manceb, Y., Frigerio, C., and Ferreira, F. 2008. "Pollen Composition and Standardization of Analytical Methods.” J. Apicult. Res. 47 (2): 156-63.

[3] Arruda, V. A. S., Pereira, A. A. S., Freitas, A. S., Barth, O. M., and Almeida-Muradian, L. B. 2013. "Dried Bee Pollen: B Complex Vitamins, Physicochemical and Botanical Composition." Journal of Food Composition and Analysis 29 (2): 100-5.

[4] Arruda, V. A. S., Pereira, A. A. S., Estevinho, L. M., and Almeida-Muradian, L. B. 2013. "Presence and Stability of B Complex Vitamins in Bee Pollen Using Different Storage Conditions." Food and Chemical Toxicology 51: 143-8.

[5] Almeida-Muradian, L. B., Pamplona, L. C., Coimbra, S., and Barth, O. M. 2005. "Chemical Composition and Botanical Evaluation of Dried Bee Pollen Pellets." Journal of Food Composition and Analysis 18 (1): 105-11.

[6] Goggi, A. S., Pollak, L., Golden, J., DeVries, M., McAndrews, G., and Montgomery, K. 2007. "Impact of Early Seed Quality Selection on Maize Inbreds and Hybrids." Maydica 52: 223-33.

[7] Kim, J. H., and Mullin, C. A. 2007. "An Isorhamnetin Rhamnoglycoside Serves as a Costimulant for Sugars and Amino Acids in Feeding Responses of Adult Western Corn Rootworms (Diabrotica virgifera virgifera) to Corn (Zea mays) Pollen.” J. Chem. Ecol. 33 (3): 501-12.

[8] Almaraz-Abarca, N., Rivera-Rodríguez, D. M., Arráez-Román, D., Segura-Carretero, A., Sánchez-González, J. J., Delgado-Alvarado, A., and Ávila-Reyes, J. A. 2013. "Pollen Phenols of the Genus Zea." Acta Bot. Mex. 105: 59-85.

[9] Ceska, O., and Styles, E. D. 1984. "Flavonoids from Zea mays Pollen." Phytochemistry 23 (8): 1822-3.

[10] Almaraz-Abarca, N., Campos, M. G., Ávila-Reyes, J. A., Naranjo-Jiménez, N., Herrera-Corral, J., and González-Valdés, L. S. 2004. "Variability of Antioxidant Activity among Honeybee-Collected Pollen of Different Botanical Origin.” Interciencia 29 (10): 574-8.

[11] Campos, M. G. 1997. "Characterization of Bee Pollen for Their Profile Phenolics and Research Some Biological Activities." Ph.D. thesis, University of Coimbra, Portugal.

[12] Campos, M. G., Mitchel, K., Cunha, A., and Markham, K. 1997. "A Systematic Approach to the Characterization of Bee Pollens via Their Flavonoid/Phenolic Profiles." Phytochem. Anal. 8: 181-5.

[13] Barth, O. M., Freitas, A. S., Oliveira, E. S., Silva, R. A.,
Maester, F. M., Andrella, R. R. S., and Cardozo, G. M. B. Q. 2010. "Evaluation of the Botanical Origin of Commercial Dry Bee Pollen Load Batches Using Pollen Analysis: A Proposal for Technical Standardization." Anais da Academia Brasileira de Ciências 82 (4): 893-902.

[14] Arruda, V. A. S., Freitas, A. S., Barth, O. M., Estevinho, M. L. M., and Almeida-Muradian, L. B. 2013. "Biological Properties of the Apiculture Coconut Pollen Collected in Northeastern Brazil.” Magistra 25: 112-74.

[15] Campos, M. G., and Markham, K. R. 2007. Structure Information from HPLC and On-line Measured Absorption Spectra: Flavone, Flavonols and Phenolic Acids, 1st ed.. Portugal: Imprensa da Universidade de Coimbra.

[16] Markham, K. R., and Campos, M. G. 1996. "7- and 8-O-Methylherbacetin-3-O-Sophoroside from Bee Pollens and Some Structure/Activity Observations." Phytochemistry 43 (4): 763-7.

[17] Markham, K. R., and Geiger, H. 1993. "1H Nuclear Magnetic Resonance Spectroscopy of Flavonoids and Their Glycosides in Hexadeuterodimethylsulfoxide." In the Flavonoides: Advances in Research Since 1986, edited by Harborne, J. B. London: Chapman \& Hall.

[18] Ou, B., Hampsch-Woodill, M., and Prior, R. L. 2001. "Development and Validation of an Improved Oxygen Radical Absorbance Capacity Assay Using Fluorescein as the Fluorescent Probe.” J. Agric. Food Chem. 49 (10): 4619-26.

[19] Zhou, T. S. 2004. An Extract of a Typhae Pollen and Its Manufacture and Use. Patent Scope WO/2004/009575, filed July 18, 2003, and issued May January 29, 2004.

[20] Chantarudee, A., Phuwapraisirisan, P., Kimura, K., Okuyama, M., Mori, H., Kimura, A., and Chanchao, C. 2012. "Chemical Constituents and Free Radical Scavenging Activity of Corn Pollen Collected from Apis mellifera Hives Compared to Floral Corn Pollen at Nan, Thailand." BMC Complement Altern Med. 12: 45.

[21] Campos, M. G., Webby, R. F., Markham, K. R., Mitchell, K. A., and Da Cunha, A. P. 2003. "Age-Induced Diminution of Free Radical Scavenging Capacity in Bee-pollens and the Contribution of Constituent Flavonoids." J. Agr. Food Chem. 51 (3): 742-5.

[22] Lopes, J., Stanciu, O. G., Campos, M. G., Almaraz-Abarca, N., Muradian, L. B., and Marghitas, L. A. 2011. "Bee Pollen Antioxidant Activity-A Review: Achievements and Further Challenges." J. of Pharmacognosy 2 (2): 25-38. 